\title{
Utricularia purpurea nuevo registro de la familia Lentibulariaceae en el Estado de Campeche, México
}

\section{New record of Utricularia purpurea of the family Lentibulariaceae in the state of Campeche, Mexico}

\author{
Ricardo Efraín Góngora-Chin*, Rodolfo Noriega-Trejo, Demián Hinojosa-Garro, Jesús Elías García-López \\ Laboratorio de Vida Silvestre y Colecciones Científicas del Centro de Estudios en Desarrollo Sustentable y Aprovechamiento de \\ la Vida Silvestre. Universidad Autónoma de Campeche. Campeche, Campeche. \\ *Autor de correspondencia: regongor@uacam.mx
}

Nota científica recibido: 12 de enero de 2015, aceptado: 15 de enero de 2016

RESUMEN. Se reporta un nuevo registro de planta vascular para el estado de Campeche que pertenece a la familia Lentibulariaceae, clasficada como Utricularia purpurea Walter, que crece en la laguna Silvituc, al sur del estado, en los municipios de Escárcega y Calakmul. Este registro sitúa al estado de Campeche con el mayor número de especies del género en la península de Yucatán.

Palabras clave: Campeche, Lentibulariaceae, península de Yucatán, Utricularia

ABSTRACT. A new record of a vascular plant classified as Utricularia purpurea Walter and belonging to the family Lentibulariaceae is reported in the State of Campeche. It grows in Silvituc lagoon, located in the southern part of the state in the municipalities of Escárcega and Calakmul. This report means that the state of Campeche now has the highest number of species belonging to this genus (6) in the Yucatan Peninsula.

Key words: Campeche, Lentibulariaceae, Yucatan Peninsula, Utricularia

\section{INTRODUCCIÓN}

La familia Lentibulariaceae está conformada por los géneros Genlisea, Pinguicula y Utricularia (Fromm-Trinta 1985, Taylor 1989, 1991, Olvera 1996, Zamudio-Ruíz 2006). Las más de 300 especies que la componen corresponden a plantas herbáceas, anuales o perennes, que pueden ser terrestres, acuáticas o epífitas (Gibson 1974, Taylor 1989, Zamudio-Ruíz 2006). Son cosmopolitas y se distribuyen en las regiones templadas, tropicales (Juniper et al. 1989) y árticas (Taylor 1989). En México se encuentran representados los tres géneros (Zamudio-Ruíz 2006), Pinguicula con 44 especies (Zamudio y Ludlow-Weichers 1993), Utricularia con 19 especies (Olvera 1996) y Genlisea con una especie (Olvera y Martínez, 2002). En Campeche se reportan las especies $U$. amethystina Salzm. ex A.St.-Hilaire \& F.Girard, U. foliosa L., U. gibba L., $U$. pusilla Vahl y $U$. subulata L. del género Utricu- laria (Carnevali et al. 2010).

El estado de Campeche conecta a la península de Yucatán con el macizo continental de México, a través del itsmo de Tehuantepec, es un área dominada por selvas fragmentadas con extensos ecosistemas inundables (Flores y Sánchez 2010). Estas condiciones favorecen una alta diversidad vegetal, debido, a la afinidad florística de estas dos regiones; además es donde existe el registro con la mayor altitud sobre el nivel del mar, lo que origina que sea el área geográfica con más diversidad de tipos de vegetación de los tres estados que conforman la península de Yucatán (Miranda y Hernández 1963, Flores-Guido y Espejel-Carvajal 1994). Sin embargo, florísticamente es el que está menos estudiado. Por lo anterior, el objetivo de este trabajo fue realizar colectas botánicas de flora acuática del estado de Campeche en sitios poco explorados, como la laguna Silvituc. 


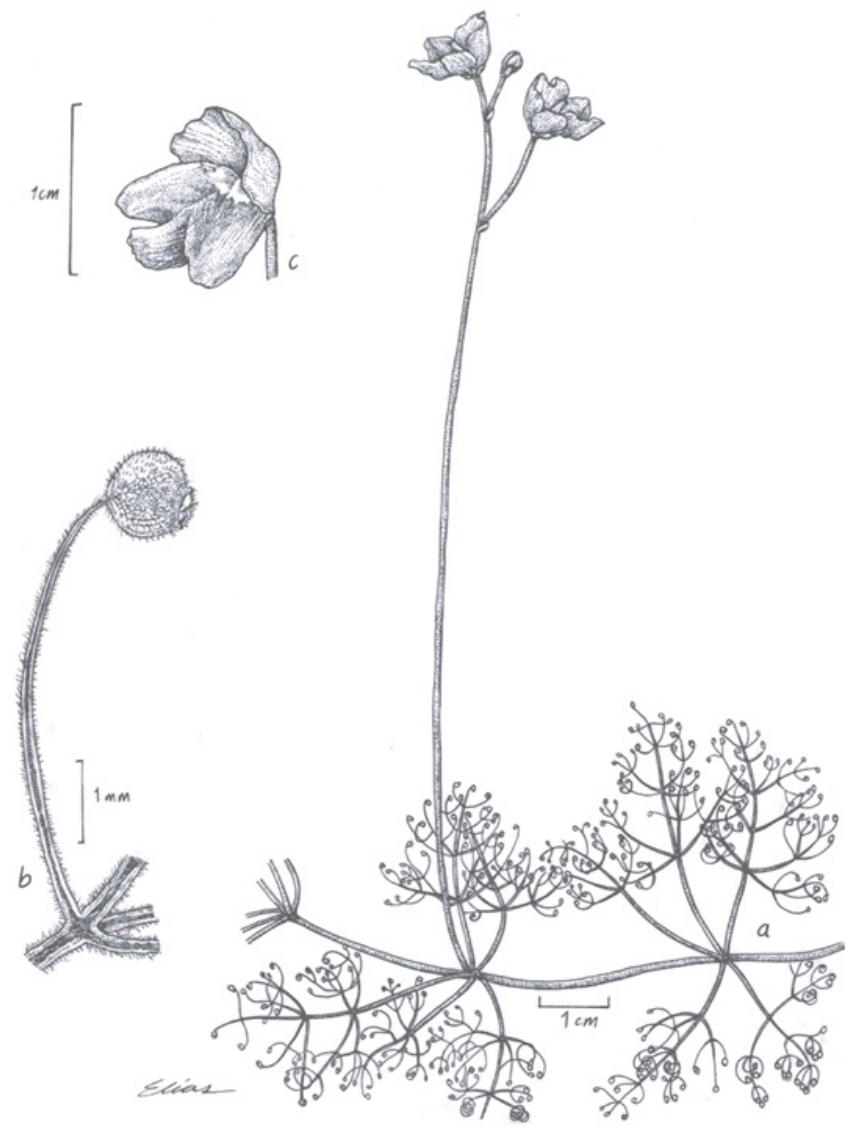

Figura 1. Utricularia purpurea. a. hábito; b. detalle de la hoja y utrículo; c. detalle de la flor. Ilustración realizada por Jesús Elías García, basada en el ejemplar de Ricardo Góngora 550 a.

\section{MATERIALES Y MÉTODOS}

El estado de Campeche se encuentra ubicado en el suroeste de la península de Yucatán (Kú, 2010). Limita al norte con el estado de Yucatán, al sur con los estados Guatemala y Tabasco, al este con el estado de Quintana Roo y al oeste con el golfo de México, entre los $17^{\circ} 49^{\prime}$ y $20^{\circ} 51^{\prime} \mathrm{LN}$ y los $89^{\circ} 06^{\prime}$ y $92^{\circ} 27^{\prime} \mathrm{LO}$, comprende los climas AW, AW1 y AW2 y el AM para la isla del Carmen (Flores-Guido y Sánchez 2010).

La laguna Silvituc se encuentra ubicada en el municipio de Escárcega, Campeche, México, es el cuerpo de agua interior más grande del estado, con coordenadas $18^{\circ} 38^{\prime} 14.73^{\prime \prime}$ LN y $90^{\circ} 17^{\prime} 05.23^{\prime \prime}$ LO, con altitud de $48 \mathrm{msnm}$, que junto con las la- gunas Las Maravillas y Chan Laguna forman el sistema hidrológico más importante del estado. Se realizaron dos salidas de colecta durante 2014, una en la época de secas y la otra en la época de lluvias, con la finalidad de colectar e inventariar la flora acuática de la laguna Silvituc, para lo cual se realizaron recorridos al azar en todo el cuerpo de agua para ubicar las especies acuáticas, de acuerdo a sus hábitos ecológicos, recolectarlas con red tipo cuchara de $10 " \times 18$ ", para tomar todo el material vegetal flotante a la deriva; y en algunos casos con pinzas de corte para hacer la separación entre especies, cuando se encontraba enredadas. Al momento de la colecta los ejemplares se montaron en prensas botánicas portátiles para su posterior secado. Los ejemplares se depositaron en el Herbario 


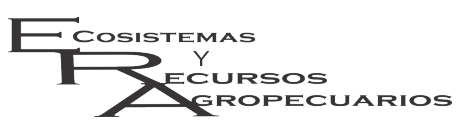

del CEDESU clave MX-HER-CAM-015-0798 de la Universidad Autónoma de Campeche.

Los ejemplares colectados se deterninaron con claves dicotómicas y literatura especializada, para el género Utricularia se empleo la clave de Olvera (1996), y se realizó la revisión de los registros para el mismo género en los herbarios del Centro de Investigación Científica de Yucatán (CICY), la Universidad Autónoma de Yucatán (UADY), el Centro de Investigaciones Históricas y Sociales (UCAM), y el herbario del Centro de Estudios de Desarrollo Sustentable y Aprovechamiento de la Vida Silvestre (CEDESU) de la Universidad Autónoma de Campeche. Para determinar que fuera Utricularia purpurea Walter, también se revisó la distribución geográfica reportada en la literatura espcializada, y se confirmó que no se encuentran ejemplares de especie en los acervos del estado de Campeche.

\section{RESULTADOS Y DISCUSIÓN}

La colecta se realizó en las coordenadas $18^{\circ}$ $37^{\prime} 07.84^{\prime \prime}$ LN y $90^{\circ} 17^{\prime} 50.51^{\prime \prime} \mathrm{LO}$, en las coordenadas $18^{\circ} 37^{\prime} 58.06^{\prime \prime}$ LN y $90^{\circ} 16^{\prime} 31.63^{\prime \prime}$ LO de la laguna Silvituc, las cuales se depositaron en el herbario del CEDESU. U purpurea es una especie que se distingue por presentar hojas verticiladas que terminan en utrículos pubescentes, la boca tiene pelos fasciculados con una glándula terminal. La corola es rosado-lila con una mancha amarilla en el centro (Figura 1). Mientras que Utricularia foliosa L., tiene flores amarillas, estolones aplanados, hojas alternas, multipinnadas con dos folíolos ovalados y desiguales en tamaño; que pueden alcanzar dimensiones de hasta $2 \mathrm{~m}$ de largo (Olvera 1996). La especie $U$. purpurea vegeta como libre flotadora en la laguna hasta una profundidad de $4 \mathrm{~m}$.

La laguna Silvituc originalmente se encontraba rodeada de selva mediana subperennifolia, selva mediana subcaducifolia y selva baja perennifolia. Pero actualmente se encuentra rodeada por cultivos de maíz, arroz, ganadería y por nucleos poblacionales, lo que ha originado un mosaico de comunidades secundarias en diferentes etapas de sucesión derivadas de estos tipos de vegetación.
Góngora-Chin et al. Utricularia purpurea en Campeche, México Esosist. Recur. Agropec. 4(10):183-186,2017

En la laguna desaguan canales provenientes de los cultivos de arroz, por lo que es común encontrar a $U$. purpurea frente a sitios de desembocaduras de corrientes proveniente de estos cultivos, y en diferentes sitios de la laguna; aunque prefiere las aguas con poco flujo. Otras especies que comparten el mismo habitat, especialmente en la orilla de la laguna son Bletia purpurea (Lam.) A.DC., Habenaria bractescens Lindl., Nelumbo lutea (Willd.) Pers., Nymphaea ampla (Salisb.) DC., Nymphoides indica (L.) Kuntze, Pachira aquatica Aubl., Typha dominguensis Pers., y diversas especies de las familias Cyperaceae y Poaceae.

En México, la distribución de Utricularia purpurea sólo se tiene reportada para los estados de Tabasco, Quintana Roo y Veracruz (Tabla 1). Esta especie se distribuye de manera natural desde Canadá hasta Centroamérica (Belice y Costa Rica) y las Antillas (Tropicos 2016). Los últimos reportes del género Utricularia para la península de Yucatán los realizaron Taylor (1989), Olvera (1996) y Carnevali et al. (2010). Con la identificación de $U$. Purpurea, el estado de Campeche se ubica como el estado con mayor número de especies del género Utricularia (seis).

Tabla 1. Distribución de Utricularia purpurea.

\begin{tabular}{lcccc}
\hline Estado & $\begin{array}{c}\text { En este } \\
\text { trabajo }\end{array}$ & $\begin{array}{c}\text { Carnevali } \\
\text { et al. }(2010)\end{array}$ & $\begin{array}{c}\text { Olvera } \\
(1996)\end{array}$ & $\begin{array}{c}\text { Taylor } \\
(1989)\end{array}$ \\
\hline Campeche & $\bullet$ &.. &.. &.. \\
Quintana Roo &.. & $\bullet$ & &.. \\
Tabasco &.. & $\bullet$ & $\bullet$ &.. \\
Veracruz &.. & $\bullet$ &.. & $\bullet$ \\
\hline
\end{tabular}

\section{AGRADECIMIENTOS}

A las autoridades ejidales y municipales del poblado Adolfo López Mateos, Silvituc, y Centenario por otorgar las facilidades para colectar los ejemplares. A la Biól. Karol Arcique Córdova quién realizó el mapa de ubicación de la laguna Silvituc y al Doctor Sergio Zamudio-Ruiz por la revisión del manuscrito. El presente trabajo se llevó a cabo con recursos del proyecto FOMIX-CAMP-172372-2011. 


\section{LITERATURA CITADA}

Carnevali G, Tapia-Muñoz JL, Duno-de Stefano R, Morillo IR (2010) Flora ilustrada de la península de Yucatán. Listado florístico. Centro de Investigación Científica de Yucatán, Mérida, Yucatán. AC. Mérida, Yucatán, México. 328p.

Flores-Guido JS, Espejel-Carvajal I (1994) Tipos de vegetación de la Península de Yucatán. Etnoflora Yucatanense. Universidad Autónoma de Yucatán, Mérida, Yucatán, México. Fascículo 3. 135p.

Flores-Guido JS, Sánchez MC (2010) Diversidad florística En: Villalobos-Zapata GJ, Mendoza Vega J (eds) La Biodiversidad en Campeche: Estudio de Estado. Comisión Nacional para el Conocimiento y Uso de la Biodiversidad (CONABIO), Gobierno del Estado de Campeche, Universidad Autónoma de Campeche, El Colegio de la Frontera Sur. México. pp: 2010-2013.

Fromm-Trinta E (1985) Lentibulariaceae do Brasil: Utricularias aquaticas. Bradea 4: 188-210.

Gibson DN (1974) Lentibulariaceae. In: Standley, PC, Williams LO, Gibson DN (eds) Flora of Guatemala. Fieldiana Bot. 24, Part X: pp: 315-328.

Juniper BE, Robins RJ, Joel DM (1989) The carnivorous plants. Academic Press, London. 353p.

Kú Quej VM (2010) Breve historia de la organización política de Campeche En Villalobos-Zapata GJ, Mendoza Vega J (eds) La Biodiversidad en Campeche: Estudio de Estado. Comisión Nacional para el Conocimiento y Uso de la Biodiversidad (CONABIO), Gobierno del Estado de Campeche, Universidad Autónoma de Campeche, El Colegio de la Frontera Sur. México. pp: 32-33.

Miranda F, Hernández X (1963) Fisiografía y vegetación. Las zonas áridas del centro y noreste de México. IMRNR. México, DF. 27p.

Olvera M (1996) El género Utricularia (Lentibulariaceae) en México. Anales del Instituto de Biología serie Botánica 67: 347-384.

Olvera GM, Martínez SE (2002) Primer registro de Genlisea (Lentibulariaceae) México. Acta Botánica Mexicana 59: 71-73.

Taylor P (1989) The genus Utricularia: a taxonomic monograph. Kew Bulletin Additional Series XIV: 1-715.

Taylor P (1991) The genus Genlisea St. Hil. Carnivorous Plant Newsletter 20: 20-35.

Tropicos (2016) Missouri Botanical Garden.http://www.tropicosorg/Name/18300098. Fecha de consulta 3 de junio de 2016.

Zamudio S, Ludlow-Wiechers B (1993) Lentibulariaceae, Pinguicula. En: Ludlow-Weichers B, Diego-Pérez N, Márquez-Guzmán J (eds). Flora Palinológica de Guerrero, No.4. Facultad de Ciencias, Universidad Nacional Autónoma de México, México, DF. 16p.

Zamudio-Ruíz S (2006) Flora del Valle de Tehuacán-Cuicatlán: Lentibulariaceae. Fasículo 45. Instituto de Biología, Universidad Nacional Autónoma de México. 10p. 